\title{
A Model-Based Approach to Measuring the Effect of Shading on Outdoor Thermal Comfort
}

\author{
Edgar Eugenio Samano Baca and I. Tsung Tsai
}

\begin{abstract}
Building shading is a tried and true strategy for passive cooling to reduce energy consumption. But shading also affects the mobility system outside of buildings. This paper analyzes the effects of building shading on outdoor thermal comfort. It focuses on building height and density as the two design parameters of urban built form and uses weather data and passive shading simulation to derive a measure of the effect of walking outdoors. The model is specialized to regions of extreme heat and humidity, drawing weather data from Abu Dhabi in the United Arab Emirates. The methodological developments are then demonstrated on four urban design schemes: high-density, low-rise; low-density, high-rise; low-density, low-rise; and high-density, high-rise. The results show that the optimal urban system for hot and humid locations, in terms of improving outdoor thermal comfort, comes from increasing the height-to-width ratio of the built urban form.
\end{abstract}

Index Terms-Shading effect, thermal comfort, thermal comfort index, urban design.

\section{INTRODUCTION}

Rapid population growth and ever-increasing urbanization rates have brought many changes to the physical environment of cities. These developments -particularly in low and middle-income regions in Africa, Asia, and Latin America, have far outpaced the rate of urban planning and controlled city growth. Furthermore, climate change and the resulting changes in temperature patterns have made a considerable impact on the health and well-being of human beings in urban dwellings. The integration of the climate dimension in city planning and design is therefore an ever more pressing issue in many of the world's metropolises.

Existing work on urban design for extreme weather conditions has been focused traditionally on cities with hot and arid climates. As an example, Fahmy and Sharples [1] used a fluid dynamics modeling tool, ENVI-met, to investigate the microclimatic thermal behavior of different urban forms in Cairo. Urban design to mitigate the negative effect of climate change in regions with hot and humid climate remains largely unexplored [2]. This paper fills this intellectual gap by analyzing the effects of shading on outdoor thermal comfort using data from Abu Dhabi, the capital of the United Arab Emirates. As population increases in urban areas and heat stress intensifies in summer, there is

Manuscript received March 1, 2014; revised May 27, 2014. This work was supported in part by Masdar Institute of Science and Technology (sponsor and financial support acknowledgment goes here) in the United Arab Emirates.

The authors are with the Institute Center for Smart and Sustainable Systems at Masdar Institute of Science and Technology, Abu Dhabi, United Arab Emirates (e-mail: ebaca@masdar.ac.ae, itsai@masdar.ac.ae). an urgent need to assess the effect of building height and density on outdoor thermal comfort under different population density schemes. A systematic and robust measure of such effect may impact policy decision-making in favor of greater walkability in urban centers.

Urban density and geometry are important urban design elements. A given urban density can result from independent design features that affect urban climate in different ways, such as: fraction of urban land covered by buildings, distances between buildings, and average height of buildings. The height of buildings is a design feature that can affect urban climate and outdoor thermal comfort in many ways. Reference [3] shows that wind speed and natural ventilation at street level are decreased when all buildings are of the same height. The height-to-width $(\mathrm{H} / \mathrm{W})$ ratio refers to the ratio between the height of buildings and the width of the streets and sidewalks that separate them. Reference [4] shows that, under low latitude conditions, the $\mathrm{H} / \mathrm{W}$ ratio and street canyon orientation has a considerable effect on solar shading and urban microclimate. As the $\mathrm{H} / \mathrm{W}$ ratio increases, the air temperature decreases; during some hours of the day, mean radiant temperature and surface temperature drop considerably [5]. The most comfortable conditions were found for narrow streets with tall buildings (higher wind speeds, lower surface temperature and meant radiant temperature).

In this paper, we focus our thermal comfort analysis on the effect of shading on pedestrian mobility patterns within the urban canopy layer to minimize thermal stress. The assessment of outdoor thermal comfort is expressed as a combination of factors that includes weather parameters -air temperature, relative humidity, wind speed, and solar radiation-, personal heat balance parameters -clothing and metabolic activity- and distance. Several attempts have been made to synthesize all related factors into a single index to define the boundaries of thermal comfort. Physiologically equivalent temperature (PET) integrates the first six and is used throughout this paper. Additional complexities, such as acclimatization and variations in eating and mobility habits, are ignored.

The remainder of this paper proceeds as follows. Section II establishes the methodology developed in this paper. Section III reframes the methodology into the context of a hot and humid region and illustrates the methodological developments on four case studies. Section IV summarizes the work and results, and proposes avenues for future work.

\section{THE MODEL}

We focus our analysis on an arbitrarily defined urban 
system as shown in Fig. 1. The symmetric urban system comprises sixteen square blocks, although only the inner 9 blocks are considered for the mobility decisions. Each block measures $m \times n(\mathrm{~m})$, and is occupied by a single building. The implication of this assumption is that the distance between buildings in each block is negligible. We consider building height and density as the design parameters of urban built form. For simplicity, it is assumed that all buildings have the same height $h(\mathrm{~m})$. Since building size is fixed for the case study presented in the next section, the difference in building density is a product of the distances between buildings. It is assumed that the distance between buildings equals the sum of the street and sidewalk width, thus making the entire block made up by a building with no front or dead spaces. Furthermore, we assume that population is evenly distributed in the specified urban system. The above assumptions allow us to examine and compare the impact of geometry alone, foregoing the complexities found in real urban textures. The attractiveness of these generic forms lie mainly in their simple and repeatable characteristics, which allows a more systematic comparative analysis on the effects of different design parameters on mobility patterns [6].

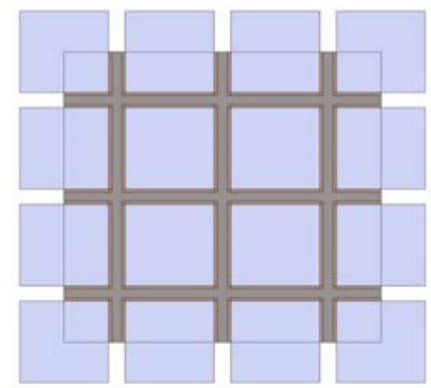

Fig. 1. Schematic of urban system.

The impact of shading on outdoor thermal comfort results from pedestrians exposed to outside weather conditions. A route decision is made as a function of distance and localized thermal comfort (indicated by a thermal comfort index that accounts for the effect of shading). Hence, each mobility decision has an associated "toll" or physical penalty.

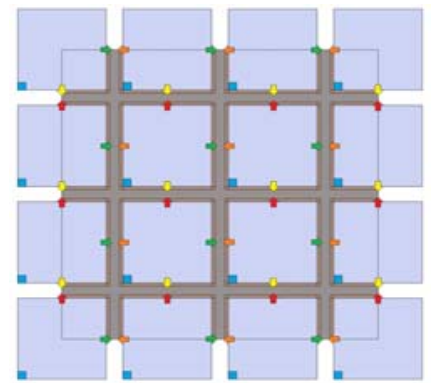

Fig. 2. Entry/exit families. Families 1, 2, 3, and 4 correspond to the red, green, yellow, and orange arrows, respectively; destinations are indicated by blue squares.

The route decision analysis is performed on an urban configuration as shown in Fig. 2. It is assumed that all potential destinations are located on the lower left corner of each block, and the access points into and out of buildings are located in the middle of each side of the buildings. It should be noted that these constraints are merely for demonstration purposes; the origins and possible destinations for each mobility event may be located anywhere along a walking path. Let entry/exit family be the access points into and out of buildings that share common route choices and shading effects; it can be found that our urban system comprises four entry/exit families as indicated by the red (entry/exit family 1 ), green (entry/exit family 2), yellow (entry/exit family 3 ) and orange (entry/exit family 4) arrows in Fig. 2. Further, it is assumed that all pedestrians are rational. The rationality assumption has three important implications in the analysis. First, pedestrians have full knowledge of available routes from each entry/exit family to their potential destinations. Second, they have full intellectual capacity to estimate the physical toll that each route would entail. Third, pedestrians will always choose the route with less physical toll, regardless of habit, a personal desire to walk longer distances, or any other external factors affecting their decisions. A final, but nontrivial assumption is that all potential destinations are equally desirable in the mind of the pedestrian.

Since a pedestrian will always choose a shorter route than a longer route that overlaps with the entire shorter route, entry/exit families 1 and 2 have three feasible route choices; whereas entry/exit families 3 and 4 have only one feasible route choice. Fig. 3 shows the three feasible route choices for entry/exit family 1 . It can be observed that the distance variation is considerable between route choices and will have a meaningful effect on an individual's decision-making. Indeed distance is the dominant factor in the decision-making of route choice under "moderate" weather conditions. On the other hand, the exposure to weather conditions will vary as well. In situations of extreme weather, individuals' choice of route is likely to be heavily influenced by the passive shading provided by the urban geometry.

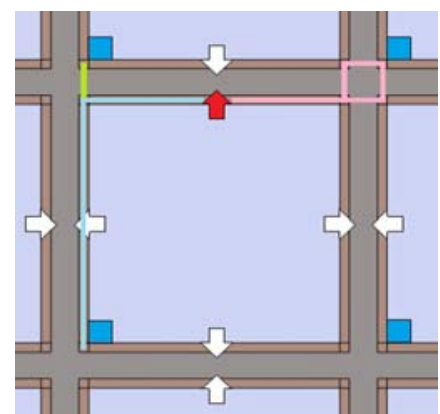

Fig. 3. Route choices for entry/exit family 1.

The calculation of route choice under extreme hot weather proceeds as follows: (1) the shading of the specified urban geometry is found using Google SketchUp. The results are given in the form of geometric shading factor s, which is the percentage of a given surface that is exposed directly to the sun at any given time. (2) The thermal comfort index of each location is calculated with the program RayMan [7]. The resolution of the thermal comfort index calculation will depend on the size of each surface that undergoes this computation. The weather and personal heat balance data is imported into the software, along with the shading values from the previous step. The result of (2) is given in the form of two common thermal comfort indices, predicted mean value (PMV) and physiologically equivalent temperature (PET). PMV treats all occupants the same and disregards adaptation to the thermal environment. PET is the air 
temperature at which the heat budget of the human body is balanced with the same core and skin temperature as under the complex outdoor conditions to be assessed in a typical indoor setting (without wind and solar radiation). In the case of both thermal comfort indices, there are six factors that affect them: clothing, metabolic rate, air temperature, solar radiation, air speed, and relative humidity. (3) Each route is evaluated in terms of physical toll, which is a function of both the distance and thermal discomfort. While the distance is determined by relative location between the entry/exit and the destination, the thermal discomfort is calculated in step (2). A heat penalty is introduced as a function of PET. Let $\alpha$ be the high temperature penalty (i.e. above a comfort temperature $t_{c}$ ) and $\beta$ the low temperature penalty (below $t_{c}$ ).

$$
\begin{gathered}
M=\alpha \max \left(t-t_{c}-3,0\right)-\beta \min \left(t-t_{c}+3,0\right) \\
N=\frac{\left|\max \left(t-t_{c}-3, d\right)+\min \left(t-t_{c}+3, d\right)-t+t_{c}-d\right|-3}{\left|t_{c}-t+d\right|-3}
\end{gathered}
$$

where $t$ is the PET at any given moment in time and $\delta$ is a small, positive scalar. The constants $\alpha$ and $\beta$ are determined by conducting sensitivity analyses (Fig. 4) so as to not dominate the effect of distance on the route decision. The heat penalty is expressed as:

$$
R_{i}=\stackrel{\circ}{J}{ }_{j=1}^{J} d e^{M}+\operatorname{In}(d) N
$$

with $j=1, \ldots, J$ number of sidewalk segments with a different shading profile. The $d$ denotes the distance walked by a pedestrian in that shading profile. $R_{i}$ is used to denote the different routes that may be taken. The first term represents the effect when temperatures are outside of the "comfort window", which is $t_{c} \pm 3^{\circ} \mathrm{C}$, and the second term represents the effect within the "comfort window", which is a function only of distance. This measure takes into account both thermal stress and walking distance.
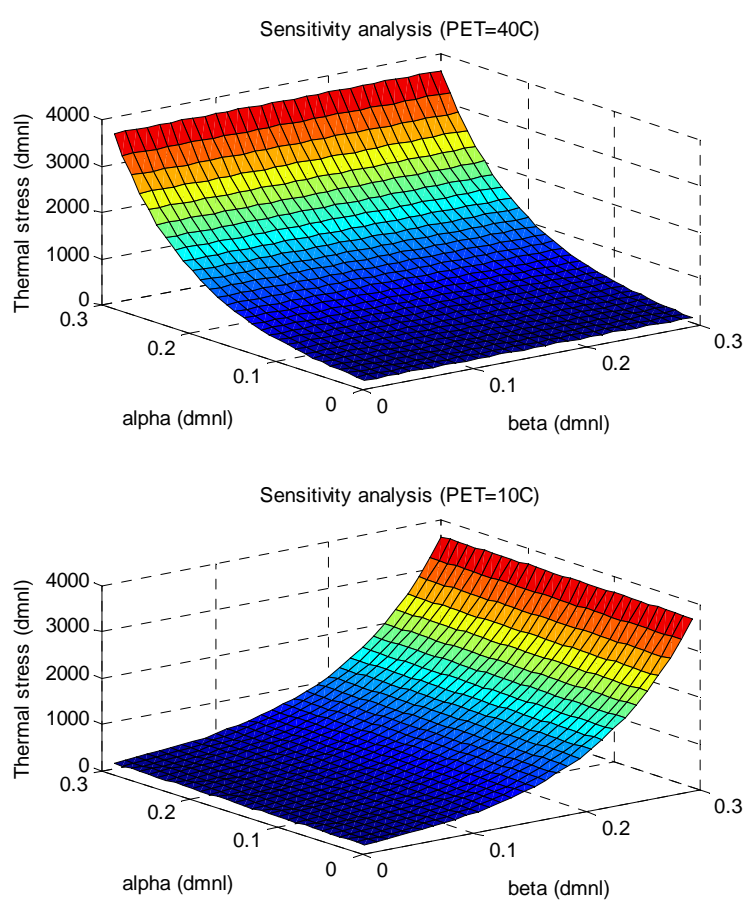

Fig. 4. Sensitivity analysis of a) $\alpha$ and b) $\beta$.

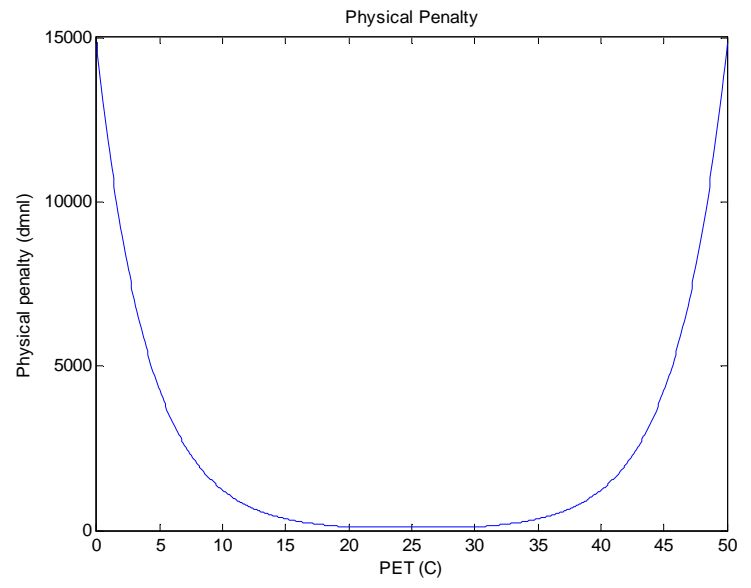

Fig. 4. Physical penalty as a function of PET.

Fig. 5 shows the physical penalty as a function of PET. Intuitively, one can imagine that the penalty is far greater for PET values exceeding the comfort temperature tc. This is due to colder temperatures being largely mitigated by the use of additional clothing, whereas the discomfort of high temperatures is difficult to avoid. Once the physical penalty values for the different routes are computed, the pedestrian's decision is expressed as:

$$
R=\min \left(R_{1}, R_{2}, \ldots, R_{I}\right)
$$

The pedestrian chooses the route that incurs a minimal amount of physical penalty to mitigate physical toll.

\section{CASE STUdy}

Table I shows the urban design principles that are analyzed using the methodology developed in the previous section. There are four schemes: the binary combinations of high and low densities, as well as high and low-rise buildings -i.e. the design parameters, building density and height, specified beforehand. Their notations should, hopefully, be easy to follow: HDLR represents high-density, high-rise; LRHD, low-density, high-rise; LDLR, low density, low-rise; and HDHR, high-density, high-rise.

For all four schemes, the same geographical location $\left(24.47^{\circ} \mathrm{N}, 54.37^{\circ} \mathrm{E}\right)$ and orientation $\left(57^{\circ}\right)$ are used -the location and orientation of Abu Dhabi.

\section{A. The Data}

The geographic data corresponds to the city of Abu Dhabi, in the United Arab Emirates, which is characterized by hot and humid weather. The personal data is fixed at average values (i.e. height of $1.72 \mathrm{~m}$ [8], weight of $72 \mathrm{~kg}$ [9], age of 42). The clothing (clo) and metabolic rate (W) are assumed constant at 0.9 clo and $80.0 \mathrm{~W}$, respectively.

The 2010 Abu Dhabi data for dry bulb temperature, solar irradiance, relative humidity, and wind speed is obtained from the National Centre of Meteorology and Seismology of the United Arab Emirates. The data has a resolution of 10 minutes.

As shown in Fig. 5, the dry bulb temperature in Abu Dhabi exhibits a clear pattern of seasonality as the mean temperature in summer is substantially higher than in winter. 
The overwhelming majority of extreme heat conditions are experienced in the months of May through October, peaking in July and August. Fig. 6 shows that global solar irradiance in Abu Dhabi stays at relatively high levels throughout April to September and thus exhibits less seasonality than the dry bulb temperature.

TABLE I: URBAN DESIGN SCHEME PARAMETERS

\begin{tabular}{|l|l|l|l|l|}
\hline Parameter & HDLR & LDHR & LDLR & HDHR \\
\hline Height $(\mathrm{m})$ & 17 & 57.8 & 17 & 57.8 \\
\hline Number of floors & $\mathrm{G}+4$ & $\mathrm{G}+17$ & $\mathrm{G}+4$ & $\mathrm{G}+17$ \\
\hline Street width $(\mathrm{m})$ & 10 & 30 & 30 & 10 \\
\hline
\end{tabular}

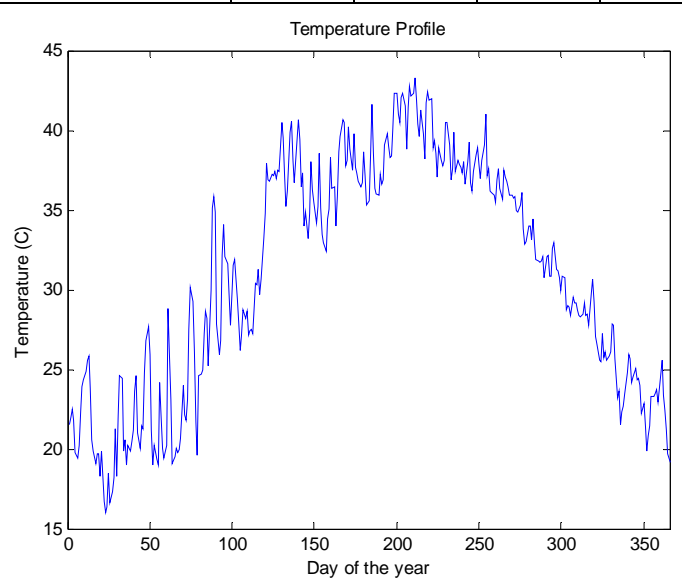

Fig. 6. Dry bulb temperature profile for Abu Dhabi in 2010. The data is shown as daily averages between 6:00 and 18:00.

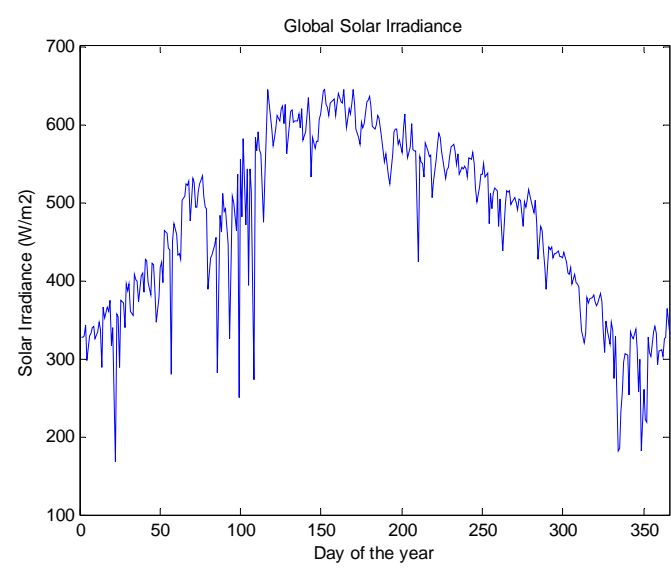

Fig. 7. Global solar irradiance profile for Abu Dhabi in 2010. The data is shown as daily averages between 6:00 and 18:00.

\section{RESULTS}

The shading factor $\mathrm{s}$-the percentage of the building's façade that is directly exposed to the sun's rays- is shown in Fig. 7 for each of the four schemes. A higher shading factor represents less shading and, thus, more exposure to solar irradiance. The seasonality is clear: $\mathrm{s}$ tends to rise in summer and fall in winter. The $\mathrm{H} / \mathrm{W}$ ratio is also a dominant variable: a larger $\mathrm{H} / \mathrm{W}$ ratio translates to a smaller shading factor. The scheme with the lowest $\mathrm{s}$ is HDHR, perhaps the least realistic of the design schemes proposed for the case study. The scheme with the largest s is LDLR, which is to be expected due to the small $\mathrm{H} / \mathrm{W}$ ratio.

Fig. 8 shows the frequency of route choice for each scheme. The route choices are numbered on ascending order of total distance walked -i.e. route 1 represents the shortest walking distance. Intuitively, this route choice is selected most of the time, while route 2 is chosen exclusively when weather conditions are most extreme and shading is relatively low. Our analysis shows that, in general, about $15 \%$ of route choices will be affected by weather conditions in all schemes. For entry/exit family 2, a probability of $2 \%$ exists for passengers to choose the longest feasible route to mitigate the physical stress from extreme hot weather.

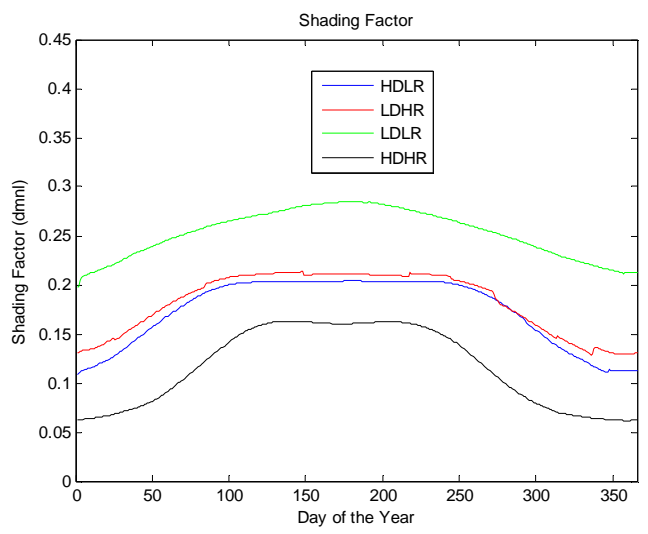

Fig. 8. Shading factor $s$ for each scheme.
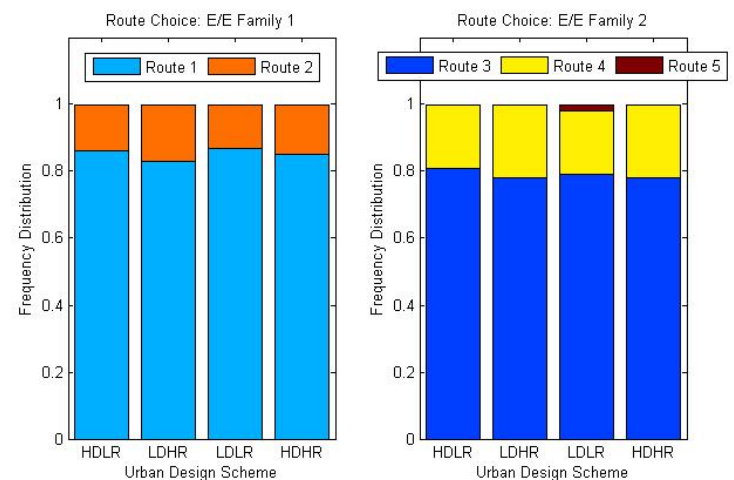

Fig. 9. Route frequencies for different case study schemes.

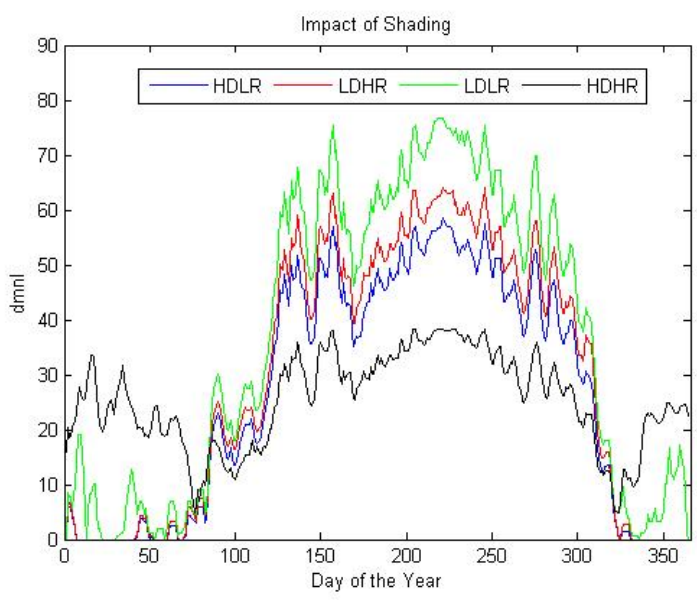

Fig. 10. Impact of shading on outdoor thermal comfort.

Fig. 9 shows the impact of shading on outdoor thermal comfort over one calendar year. This figure is shown as the reciprocal relative to the physical toll if weather conditions are ideal -i.e. the effect of distance is eliminated from Eq. (3). This translates to higher values representing a larger loss of thermal comfort. It is worthwhile to note that it hits its peak over the summer months, cresting between July and August. These are, not so coincidentally, the months of most extreme 
temperatures. The actual values of the measure are dimensionless and have little absolute meaning. For example, when comparing the four design schemes proposed in the case study, it yields interesting insights related to the outdoor thermal comfort of pedestrians.

\section{DISCUSSION}

Among the urban design elements, archetypal urban form, density, and geometry have important implications in matters of urban planning, such as built potential, daylighting, passive cooling potential, and walkability. The sustainable performance of urban systems is measured upon these and many other factors. The question of which urban design performs best cannot have an absolute answer. When a large number of variables are taken into account, it is likely that conflicts will emerge amongst them; terms such as "best" and "optimal" will certainly embody value judgments. One thing that is certain, however, is the need to understand the principles and dynamics that govern such variables. Only then can the entirety of their implications -and potential benefits- be used for the betterment of the society around them.

The concept of a measure of the impact of shading on outdoor thermal comfort draws questions about the value of this measure. Why is it even necessary? To this end, it is important to recognize that this measure can be associated with quantifiable measures of cost and benefit.

In the building domain, this measure can be associated with daylighting, heat exchange, and passive cooling. These have important implications on energy consumption and greenhouse gas emissions. In the mobility domain, this measure can be associated with health, productivity, and walkability. These have important implications on health, societal equality, and economic output. The three conventionally accepted pillars of sustainability -society, economy, and environment- can all be associated, and potentially benefit from, the methodological developments presented in this paper.

Lastly, the methodological developments outlined in this paper address a clear gap in knowledge between the climatic conditions of urban canopies and their effect on the individuals that dwell among them.

\section{CONCLUSION}

This paper has shown that it is possible to model and measure the impact of shading on outdoor thermal comfort. This measure can be used to yield valuable insights pertaining to existing systems, as well as a tool for long-term planning and decision-making.

Abu Dhabi weather data was used to quantitatively analyze the effect of shading on an urban mobility system. Four urban design schemes were analyzed: high-density, low-rise; low-density, high rise; low-density, low-rise; and high-density, high-rise. The results show a clear effect of shading on the thermal stress levels of pedestrians. LDHR and HDLR, because of their similar H/W ratios, exhibit similar levels of thermal stress throughout the year. The results are sensitive to weather data; however, this is not the dominant independent variable. Distance seems to be equally as important, and more so during more moderate climatic conditions. This fact is confirmed by field studies that suggest most people will generally take the shorter route to their destination -often due to time constraints or habit, both decision variables that were not explicitly considered in this analysis.

As demonstrated in the above case study, the specification of the climatic zone within which any environmental research on urban systems is taking place is fundamental in the analysis of the results. Finally, the primary interest was in analyzing the impact of urban geometry. Many parameters have not been taken into account directly in the analysis.

In future work, the effect of orientation on the thermal comfort of pedestrians ought to be analyzed. Additionally, the authors seek to develop a measure of productivity as a function of outdoor thermal comfort. Analyzing the impact of shading on the energy consumption of buildings is another challenging avenue of future work. Finally, integrating all these measures into a single index might provide a decent approximation of the value proposition of shading in an urban system.

\section{REFERENCES}

[1] M. Fahmy and S. Sharples, "Passive design for urban thermal comfort: a comparison between different urban forms in Cairo, Egypt," in Proc. the $25^{\text {th }}$ Conference on Passive and Low Energy Architecture, Dublin, 2008, pp. 1-6.

[2] R. Emmanuel, "Thermal comfort implications of urbanization in a warm-humid city: the Colombo metropolitan region (CMR), Sri Lanka," Buildings and Environment, vol. 40, pp. 1591-1601, 2005.

[3] E. Johansson and R. Emmanuel, "The influence of urban design on outdoor thermal comfort in the hot humid city of Colombo, SRI Lanka," International Journal of Biometeorology, vol. 51, pp. 119-133, 2006.

[4] F. Bourbia and H. Awbi, "Building cluster and shading in urban canyon for hot dry climate Part 2: Shading simulations," Renewable Energy, vol. 29, no. 2, pp. 291-301, 2004.

[5] A. N. Kakon, M. Nobuo, S. Kojima, and T. Yoko, "Assessment of thermal comfort in respect to building height in a high-density city in the tropics," American Journal of Engineering and Applied Sciences, vol. 3, no. 3, pp. 545-551, 2010.

[6] C. Ratti, D. Raydan, and K. Steemers, "Building form and environmental performance: archetypes, analysis and an arid climate," Energy and Buildings, vol. 35, pp. 49-59, 2003.

[7] A. Matzakaris and C. Endler, "Physiologically equivalent temperature and climate change in Freiburg," Meteorological Institute, Freiburg.

[8] J. R. Cameron, J. G. Skofronick, and R. M. Grant, Physics of the Body, 2nd ed., United States: Wiley, 1999.

[9] S. C. Walpole, D. Prieto-Merino, P. Edwards, J. Cleland, G. Stevens, and I. Roberts, "The weight of nations: an estimation of adult human biomass," BMC Public Health, vol. 12, no. 439, 2012.

[10] 2013 ASHRAE Handbook - Fundamentals, Tech. Rep., ASHRAE, Atlanta, USA, 2013.

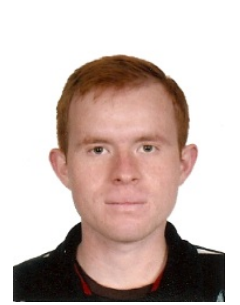

Edgar Eugenio Samano Baca was born in Mexico City, Mexico, in 1987. He received his bachelor's degree in mechatronics engineering from Universidad de Las Americas Puebla (UDLAP), Mexico, in 2009. He is currently working towards his master's degree in the engineering systems and management program at Masdar Institute of Science and Technology in Abu Dhabi, United Arab Emirates.

$\mathrm{He}$ has worked as a research assistant at the Instituto Nacional de Astrofisica, Optica y Electronica (INAOE), Consiglio Nazionale delle Ricerche (CNR), and Masdar Institute of Science and Technology. He completed the rescue and rehabilitation of public spaces campaign as project manager, and was a part-time professor at the University level for one year. 
His current research interests include transportation, energy systems, and modeling and control of complex systems.

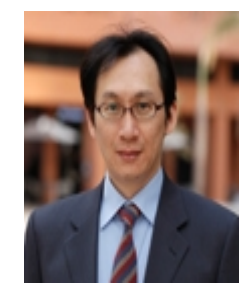

I. Tsung Tsai received a master and doctorate degrees in massachusetts institute of technology (MIT). Now he is an assistant professor in engineering systems and management program in Masdar Institute of science and Technology.
Prior to joining Masdar Institute Faculty, Dr. I. Tsung Tsai served as a water strategy consultant for the Singapore government and was a visiting scholar to the Institute of Financial Research and Management, India in 2006. His research interests include carbon management, carbon finance, infrastructure policy and information economics. His current research estimates the effect of emission allowances on human health. Previously, he studied the implicit incentive mechanism embedded in financial contracts, and the effect of asymmetric information in the online auction markets. 